\title{
Disruption of GABA shunt affects Trichoderma atroviride response to nutritional and environmental stimuli
}

\author{
Luboš Nižnanskýa, b, L'udovít Varečka ${ }^{\text {b }}$, Svetlana Kryštofováb \\ ${ }^{a}$ Institute of Forensic Medicine, Faculty of Medicine, Comenius University in Bratislava \\ ${ }^{b}$ Institute of Biochemistry and Microbiology, Faculty of Chemical and Food Technology, \\ Slovak University of Technology in Bratislava \\ svetlana.krystofova@stuba.sk
}

\begin{abstract}
The fungus Trichoderma atroviride is a member of the genus Trichoderma to which belong many species known for high cellulase production, formation of various antibiotics, plant biocontrol and antagonistic activities against other fungi. Deletion of T. atroviride glutamate decarboxylase gene gad caused minor defects in germination, hyphal branching, slower growth and disruption of conidiation pattern. GABA can be used by fungi as a secondary carbon source and as a primary nitrogen source. We analyzed the effect of different nutrient compositions and environmental conditions (light and temperature) on growth and development of T. atroviride in strains defective in the functional GAD. The gad mutants grown on $\mathrm{NH}_{4} \mathrm{NO}_{3}$ as a sole carbon source grew slower and formed conidiation bands closer to each other which was clearly demonstrated during their cultivation in race tubes. The gad mutants exhibited slightly lower apical extension growth rate at the room temperature but their apical extension rate dropped significantly at $30^{\circ} \mathrm{C}$. Higher temperature had also inhibitory effect on $\mathrm{gad}$ mutant conidiation, whereas $30^{\circ} \mathrm{C}$ seems optimal temperature for the parental strain. The optimal temperature for $\mathrm{gad}$ mutant conidiation was lower than in $\mathrm{F} 534$, about $25^{\circ} \mathrm{C}$.
\end{abstract}

Keywords: Trichoderma, GABA shunt, carbon and nitrogen metabolism, development

\section{Introduction}

Trichoderma species (ascomytes) belong to soil free living microorganisms whose genome sequences indicate that Trichoderma strains could have ability to live as phytopathogens but during evolution they evolved to cohabitate with plants on friendly terms and are stabile associates of rhizoid sphere (Druzhinina et al., 2011). Trichoderma has been successfully used as biopesticide and biocontrol agent in agriculture and forestry (Harman et al., 2004a). Some Trichoderma isolates can degrade organic chemicals, chlorophenolic compounds and xenobiotic pesticides (Harman et al., 2004b). Although commercial preparations of Trichoderma spp. for biological control or cellulose production requires strains with high numbers of conidia (asexual spores), good biocontrol activity and high cellulose secretion relies upon the fungus being metabolically active. Understanding the factors that regulate the morphogenic changes between vegetative and dormant stages of Trichoderma life cycle remains high priority for scientific community. The transition from fungal mycelium to conidiation is the result of multiple environmental stimuli, whereby one factor is not always sufficient to induce the morphological switch. One of the known stimuli influencing conidiation and vegetative growth in Trichoderma is C:N (carbon : nitrogen) status (Steyaert et al., 2010 a). Generally, carbon and nitrogen metabolisms are interconnected. One such connection leads to the GABA shunt (Fig. 1). The role of GABA in life cycle of filamentous fungi remains still elusive (Kumar and Punekar, 1997). GAD has been previously purified (Hao and Schmit, 1991) and its expression determined in conidiating cultures (Hao and Schmit, 1993). In Trichoderma atroviride, it has been demonstrated that GAD was developmentally regulated and no activity was detected in the nonconidiating strain (Strigáčová et al., 2001). These changes were caused by transcriptional regulation of the gad gene in both submerged mycelium and aerial hyphae after the light exposure (Pokorný et al., 2005). Furthermore, deletion of Trichoderma atroviride gad genes has led to reductions in respiration, biomass accumulation and changes in conidiation pattern. Recent studies also indicated that GAD positive regulation could be governed by calcineurin signaling pathway (Nižnanský et al., 2013). The gad genes were identified and characterized in Aspergillus nidulans (Ray et al., 2004) and in Aspergillus oryzae (Kato et al., 2002). GAD has been shown to participate in the regulation of metabolism under hypoxic conditions (Masuo et al., 2010). Studies with Fusarium graminearum demonstrated that GABA shunt plays a role in plant-fungus interaction and in fungal metabolism during pathogenesis (Carapito et al., 2008, Bönnighausen et al., 2015). In the present study, we investigated possible role of GABA metabolism 
in $T$. atroviride growth and conidial development as well as the effect of GABA loss on growth and conidiation under different culture conditions.

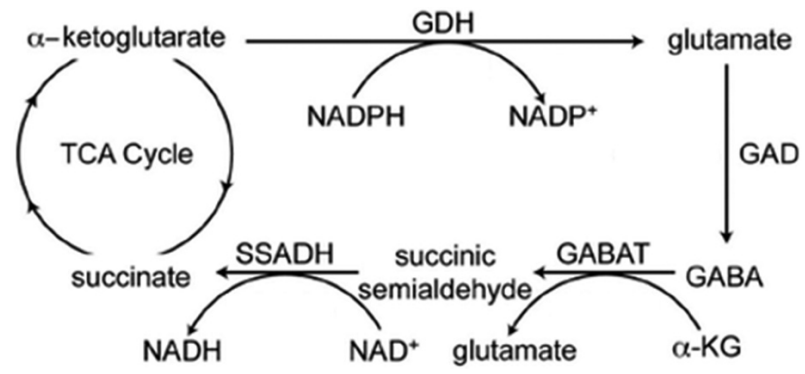

Fig. 1. GABA shunt. L-glutamate is formed by transamination of 2-oxoglutarate (KG) from the tricarboxylic acid (TCA) cycle. The reaction is catalyzed by the mitochondrial glutamate dehydrogenase. L-glutamate is decarboxylated by cytosolic GAD (glutamate decarboxylase) to GABA which is transported into mitochondria where is deaminated by GABAT (GABA transaminase) to succinate semialdehyde. The last enzyme of GABA shunt is succinate semialdehyde dehydrogenase (SSADH) which catalyzes succinate semialdehyde oxidation to succinate. The latter compound enters the TCA cycle and GABA can be resynthesized from 2-oxoglutarate.

\section{Methods}

The T. atroviride CCM F534 was obtained from the Czech Collection of Microorganisms in Brno, Czech Republic. All strains were stored as $20 \%$ glycerol conidia stocks at $-70{ }^{\circ} \mathrm{C}$. The strains were maintained on modified Czapek-Dox medium (CZD) $\left(\mathrm{g} \cdot \mathrm{L}^{-1}\right)$ : sucrose (30), $\mathrm{MgSO}_{4} \cdot 7 \mathrm{H}_{2} \mathrm{O}(1), \mathrm{FeSO}_{4}$. $7 \mathrm{H}_{2} \mathrm{O}(0.01), \mathrm{KCl}(0.5), \mathrm{K}_{2} \mathrm{HPO}_{4}(1)$, agar (20), pH 6.7 supplemented with one of the following nitrogen sources: $\mathrm{NH}_{4} \mathrm{NO}_{3}\left(2 \mathrm{~g} \cdot \mathrm{L}^{-1}\right), \mathrm{NaNO}_{3}\left(1,8 \mathrm{~g} \cdot \mathrm{L}^{-1}\right)$, GABA $\left(2.6 \mathrm{~g} \cdot \mathrm{L}^{-1}\right)$. In those cases when sucrose was replaced by GABA, its concentration was identical to sucrose $\left(88 \mathrm{mmol} \cdot \mathrm{L}^{-1}\right)$. If not indicated otherwise, strains were grown on solid CZD at $27{ }^{\circ} \mathrm{C}$ in $12 \mathrm{~h}$ light/dark conditions. Conidia were collected from 10-day old cultures grown on CZD agar medium with $\mathrm{NH}_{4} \mathrm{NO}_{3}$ into sterile water, filtered through three layers of gauze and concentrated by centrifugation at $2500 \mathrm{~g}$. The conidial suspension was subsequently used to inoculate agar plates. At least two independent gad deletion mutants were assayed on agar plates with at least two technical replicates.

\section{Results and discussion}

The type of carbon source and its concentration has been suggested as the primary factor in the both dark and light-induced conidiation in T. viride and T. atroviride (Chovanec et al., 2001, Friedl et al., 2008). T. viride cultures were able to grow on 30 out of 32 carbon sources, including polysaccharides, amino acids and alcohols. Hexoses and disaccharides, but not pentoses and amino acids, promoted both growth and conidiation induced by aging or light. When sucrose as a sole carbon source and $\mathrm{NH}_{4} \mathrm{NO}_{3}$ as a sole nitrogen source were used in culture media, deletion of $\mathrm{gad}$ in $T$. atroviride CGM F534 (formerly T. viride) led to lower apical extension growth rates and subsequently shorter distances between formed conidial bands either on large plates (Nižnanský et al., 2013) or in race tubes (Fig. 2). However, the strains grown on the same medium augmented with sucrose demonstrated slightly different conidiation pattern on smaller plates (9 cm in diameter) caused by spatial growth restriction. Parental strain F534 formed two circular conidial bands: one triggered by the light and the second one at the plate perimeter (Fig. 3). The race tubes experiment design to observe both growth rates and conidial band formation over longer period of time clearly showed differences between parental and gad deletion strains which might be sometimes harder to determine during cultivation in small Petri dishes where full conidial rings sometimes fail to be fully formed. These data also uncovered some important differences between GABA metabolism of filamentous fungi and yeasts. Deletion of yeast UGA1 (GABA aminotransferase) and GAD1 (glutamate decarboxylase) lengthened the lifespan (Kamei et al., 2011) but gad deletion in T. atroviride has only shorten the distance between conidiation bands due to slower growth.

Sucrose replacement by GABA in CZD medium as a sole carbon source significantly suppressed growth and conidiation of both parental and gad mutant strains (Fig. 3). Furthermore, gad also demonstrated significantly longer lag phase during submerged cultivation (Nižnanský et al., 2013) proving GABA to be a poor carbon source for biomass accumulation and conidia production. When sucrose and GABA together were used as carbon source the conidiation did not differ from that observed on CZD medium with sucroce alone (Fig. 3). Although, GABA serves as a good nitrogen source to many fungi, $\mathrm{NH}_{4} \mathrm{NO}_{3}$ replacement by GABA in culture medium led to suppression of conidiation and formation of conidiation rings was inhibited in both parental strain and gad mutants (data not shown). The nitrogen status has been shown to cross-regulate conidiation (Ellison et al., 1981, Steyaert et al., 2010 b). In the presence of preferred (primary) nitrogen sources, organisms repress expression of genes required for the utilization of secondary sources and this process is termed 


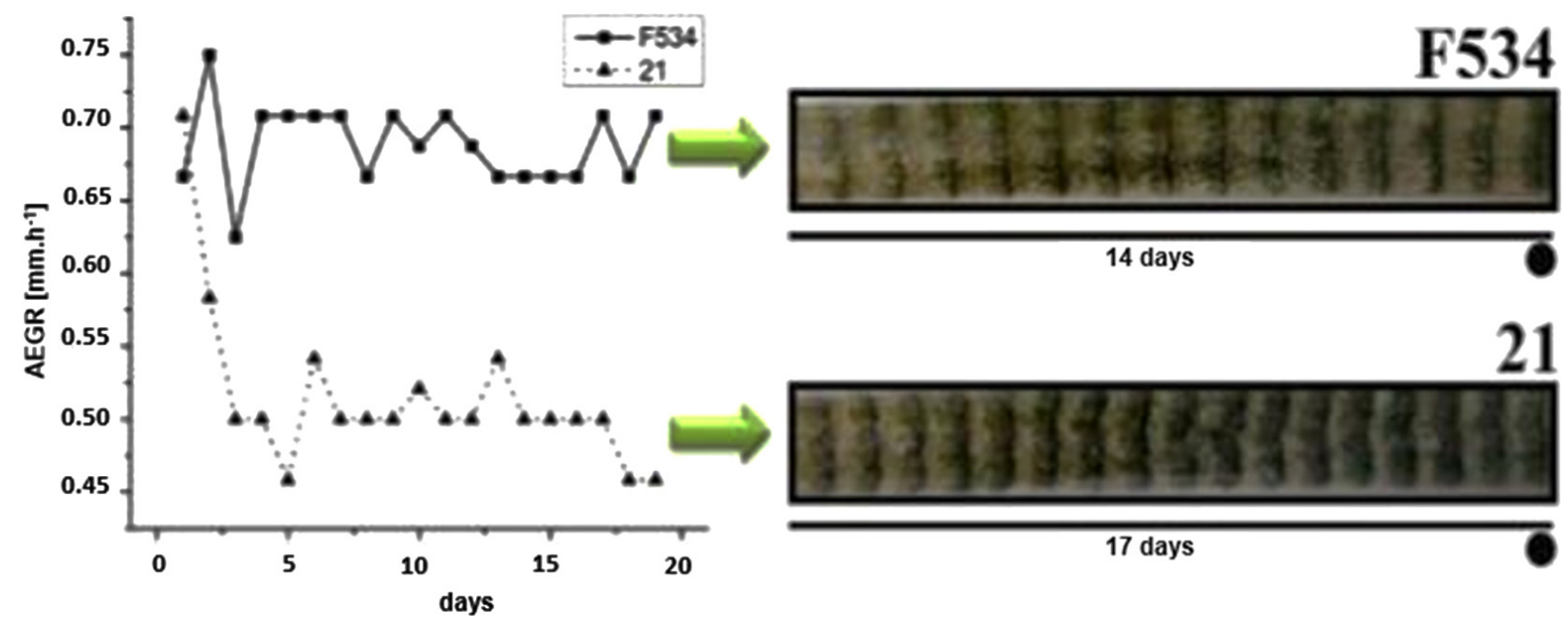

Fig. 2. The effect of gad mutation on apical extension growth rate (AEGR, the right diagram) and conidial band formation (the left photograph). The parental strain F534 and $\Delta$ gad mutant strain 21 were grown on modified CZD medium containig $\mathrm{NH}_{4} \mathrm{NO}_{3}$ as a sole nitrogen source and sucrose as a carbon source in circadian conditions ( $12 \mathrm{~h}$ in the light and $12 \mathrm{~h}$ in the dark) at $27^{\circ} \mathrm{C}$ for 25 days.

The represenative experiment of three is shown.

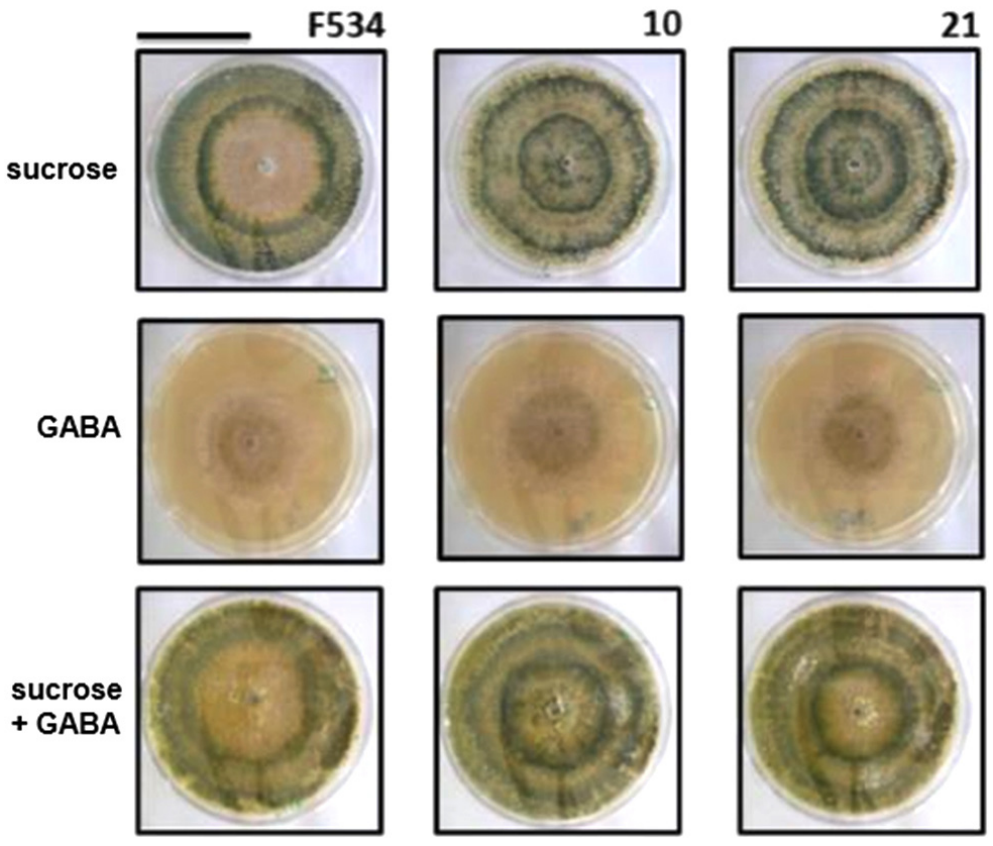

Fig. 3. The effect of various carbon sources on conidiation pattern of the parental strain F534 and $\mathrm{gad}$ mutants 10 and 21 grown in modified CZD medium containig $\mathrm{NH}_{4} \mathrm{NO}_{3}$ as a sole nitrogen source and either sucrose and/or GABA as sole carbon sources. Strains were grown in circadian conditions $(12 \mathrm{~h}$ in the light and $12 \mathrm{~h}$ in the dark) at $27^{\circ} \mathrm{C}$ for 12 days. The represenative experiment of three is shown. The bar indicates $5 \mathrm{~cm}$.

as nitrogen catabolite repression (NCR). When primary sources are low and secondary sources are high, derepression occurs. Amino- and ammoniumderived primary nitrogen sources are frequently used to induce NCR in filamentous fungi, and growth on $\mathrm{KNO}_{3}$ or other nitrates strongly induces nitrogen derepression (Marzluf, 1997, ter Schure et al., 2000). By supplementing culture media with single nutri- ents present in the richer media, primary forms of nitrogen can convert a peripheral sporulation to a concentric one and the uptake of primary nitrogen in a cell must be sufficient to allow simultaneous growth and conidiation (Ellison et al., 1981).

Trichoderma spp. frequently grow in soil in dark environment and different climate regions. Therefore, we examined effect of three different temperatures 


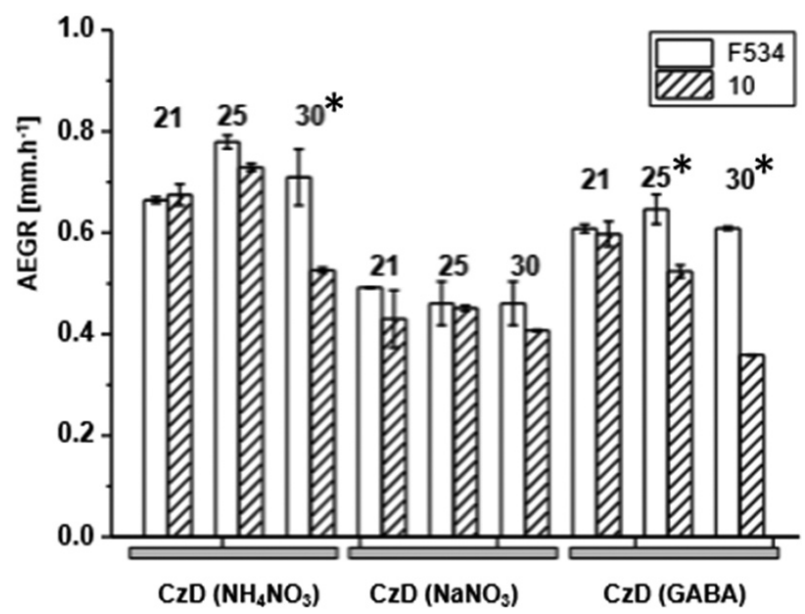

Fig. 4. The effect of temperature and various nitrogen sources on apical extension growth rate (AEGR) of the parental strain F534 and $\Delta$ gad mutant strain 10. The strains were grown on modified CZD medium supplemented with

$\mathrm{NH}_{4} \mathrm{NO}_{3}, \mathrm{NaNO}_{3}$ and GABA at 21, 25 and

$30^{\circ} \mathrm{C}$ in the dark. Representative of two experiments is shown. Statistical significance was defined as a two-tailed $\mathrm{P}$ value of $<0.001$. AEGRs with statistical differences are marked by an asterix.
21, 25 and $30^{\circ} \mathrm{C}$ on growth and conidiation in the dark. Simultaneously, we used three different nitrogen sources to examine their effect on apical extension growth rates (Fig. 4). Growing strains at room temperature and on $\mathrm{NaNO}_{3}$ as a nitrogen source did not result in significant differences in apical extension growth rate between the parental strain and mutants. However, growth at higher temperature of $30^{\circ} \mathrm{C}$ and on primary nitrogen sources $\left(\mathrm{NH}_{4} \mathrm{NO}_{3}\right.$ and GABA) caused up to $30 \%$ decrease in apical extension rate in gad mutants in comparison to F534. The effect of various temperatures on conidiation in the dark differed significantly between F534 and gad mutants (Fig. 5). The parental strain produced narrow conidial rings at the plate periphery at 21 and $25^{\circ} \mathrm{C}$ and much wider conidiation ring at $30{ }^{\circ} \mathrm{C}$ indicating optimal temperature for conidiation closer to $30{ }^{\circ} \mathrm{C}$. Contrary to F534, gad mutants formed very thin conidiation ring in the center of plates at $30^{\circ} \mathrm{C}$ and very wide conidiation rings at the plate periphery at 21 and $25^{\circ} \mathrm{C}$ indicating optimal temperature for mutant conidiation between 21 and $25{ }^{\circ} \mathrm{C}$. GABA deletion has caused thermosensitivity in rendered gad mutants and the temperature optimum was shifted to lower temperature. This finding is similar to that demonstrated in Saccharomyces cerevisiae in which was GABA shunt responsible for thermotolerance (Cao et al., 2013).
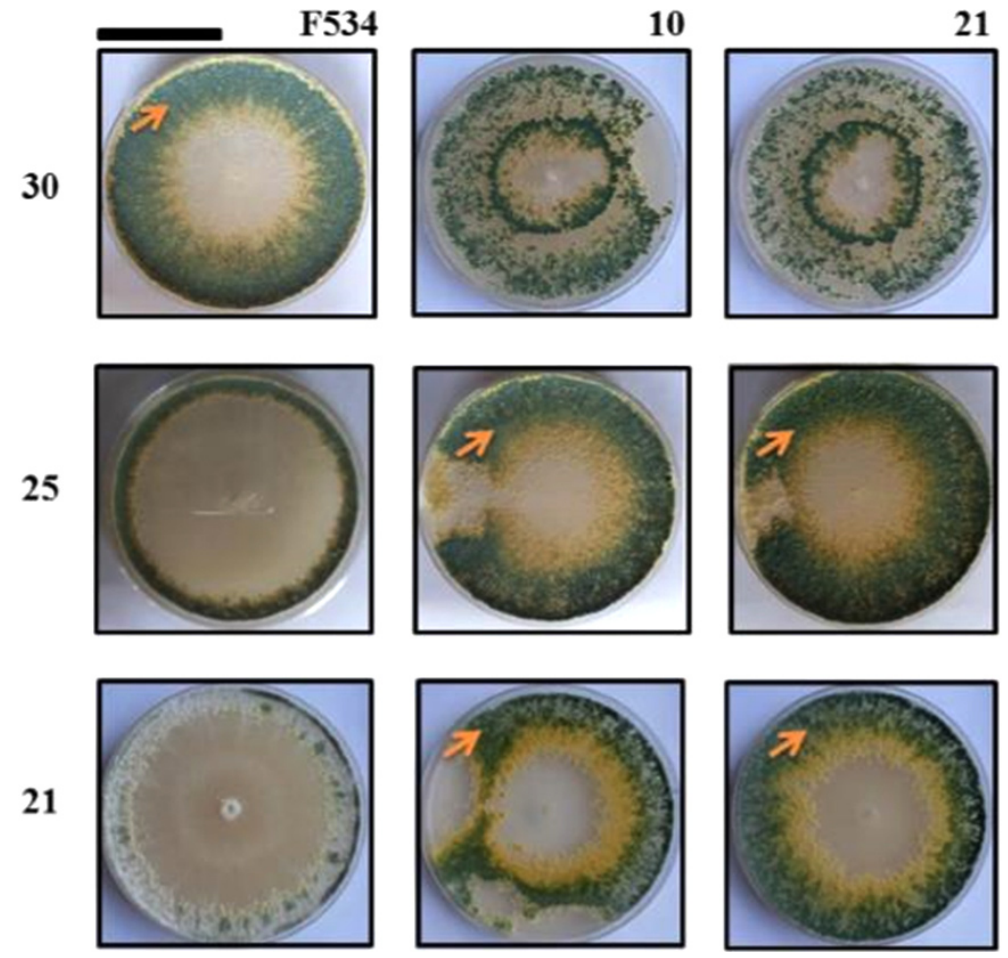

Fig. 5. The effect of temperature on conidiation of the parental strain F534 and gad mutants 10 and 21 grown in modified CZD medium containig $\mathrm{NH}_{4} \mathrm{NO}_{3}$ as a sole nitrogen source and sucrose as a sole carbon source at three different temperatures 21,25 and $30^{\circ} \mathrm{C}$ in the dark for 7 days.

The represenative experiment of three is shown. The bar indicates $5 \mathrm{~cm}$. 
To summarize the effect of GABA shunt on $T$. atroviride development and physiology, we concluded that disruption of this metabolic pathway did not have any detrimental influence on phenotype at standard laboratory conditions. However, the aberration in gad mutant growth and conidiation could place the defective strains in disadvantage while living in harsh, competitive environment with limited resources.

\section{Acknowledgement}

This work was supported by courtesy of the Slovak Grant Agency VEGA 1/0870/14, the Slovak Research and Development Agency APVV-0719-12, APVV/0282-10, and by EU project ITMS 26240120028.

\section{References}

Bönnighausen J, Gebhard D, Kröger C, Hadeler B, Tumforde T, Lieberei R, Bergemann J, Schäfer W, Bormann J (2015) Mol. Microbiol. 98(6): 1115-32.

Cao J, Barbosa JM, Singh NK, Locy RD (2013) Yeast 30(4): 129-44.

Carapito R, Hatsch D, Vorwerk S, Petkovski E, Jeltsch J-M, Phalip V (2008) Fungal Genet. Biol. 45: 738-748.

Chovanec P, Hudecová D, Varečka L (2001) Folia Microbiol 46(5): 417-22.

Druzhinina IS, Seidl-Seiboth V, Herrera-Estrella LR, Horwitz BA, Kenerley CM, Monte E, Mukherje PK, Zeilinger S, Grigoriev I., Kubicek CP (2011) Nature 9: 749-759.

Ellison PJ, Harrower KM, Chilvers GA, Owens JD (1981) Trans. Br. Mycol. Soc. 76: 441-445.
Friedl, MA, Kubicek CP, Druzhinina IS (2008) Appl. Environ. Microbiol. 74: 245-250.

Harman GE, Howell CR, Viterbo A, Chet I, Lorito M (2004a) Nat. Rev. Microbiol. 2: 43-56.

Harman GE, Lorito M, Lynch JM (2004b) Adv. Appl. Microbiol. 56: 313-330.

Hao R, Schmit JC (1993) Biochem. J. 293(3): 735-738.

Hao R, Schmit JC (1991) J. Biol. Chem. 266: 5135-5139.

Kamei Y, Tamura T, Yoshida R, Ohta S, Fukusaki E, Mukai Y (2011) Biochem Biophys Res Commun. 407(1): 185-90.

Kato Yuki, Kato Yoko, Furukawa K, Hara S (2002) Biosci. Biotechnol. Biochem. 66: 2600-2605.

Kumar S, Punekar NS (1997) Mycol. Res. 101(4): 403-409.

Masuo S, Terabayashi Y, Shimizu M, Fujii T, Kitazume T, Takaya N (2010) Mol Genet Genomics 284: 415-424.

Marzluf GA (1997) Microbiol. Mol. Biol. Rev. 61: 17-32.

Nižňanský L, Kryštofová $S$, Vargovič $P$, Kaliňák M, Simkovič M, Varečka L (2013) Antonie Van Leeuwenhoek 104(5): 793-807.

Pokorný R, Vargovič P, Hölker U, Janssen M, Bend J, Hudecová D, Varečka L' (2005) J. Basic Microbiol. 45: 219-229.

Ray A, Macwana S, Ayoubi P, Hall LT, Prade R, Mort AJ (2004) BMC genomics 5: 22.

Steyaert JM, Weld RJ, Mendoza-Mendoza A, Stewart A (2010a) Microbiology (Reading, England) 156: 2887-900.

Steyaert JM, Weld RJ, Stewart A (2010b) Fungal Biol. 114: 179-188.

Strigáčová J, Chovanec P, Liptaj T, Hudecová D, Turský T, Šimkovič M, Varečka L' (2001) Arch. Microbiol. 175: 32-40.

ter Schure EG, van Riel NAW, Verrips CT (2000) FEMS Microbiol. Rev. 24: 67-83. 